\title{
Stable multi-wavelength erbium fiber ring laser with optical feedback for remote sensing
}

\author{
S. Diaz, D. Leandro, and M. Lopez-Amo, Senior Member, IEEE
}

\begin{abstract}
In this work, we demonstrate a stable fiber sensing system for remote temperature measurements, where the sensing element is an array of four fiber Bragg gratings (FBGs) and sensor interrogation is achieved with a multi-wavelength erbium fiber ring laser. By introducing a feedback fiber loop in a fiber ring cavity, four laser emission lines were obtained simultaneously in single-longitudinal mode operation (SLM). The power instability obtained was lower than $0.5 \mathrm{~dB}$ with an optical signal-to-noise ratio (OSNR) higher than $50 \mathrm{~dB}$ for all the emitted wavelengths. The application of this system for remote temperature measurements has been demonstrated even though the SLM regime cannot be preserved.
\end{abstract}

Index Terms - Erbium-doped fiber (EDF), fiber Bragg grating (FBG), multi-wavelength lasing, single-longitudinal-mode (SLM).

\section{INTRODUCTION}

$\mathrm{M}$ ULTI-WAVELENGTH fiber lasers have attracted considerable interest because of their potential applications in dense wavelength division multiplexing (DWDM), optical fiber sensing, optical instrumentation, and photonic generation of microwave signals. Several approaches have been employed to implement multiwavelength erbiumdoped fiber-ring lasers (MEDFRLs) such as short linear cavity distributed Bragg reflector fiber lasers [1], fiber distributed feedback lasers [2] and fiber ring lasers with different ringcavity configurations [3-5].

Among these schemes, a number of fiber ring lasers have been specially studied and demonstrated. However, the fiber ring laser unavoidably generates an enormous number of densely spaced longitudinal modes lying beneath the erbium gain profile due a rather long cavity length. Thus, fiber ring lasers usually perform unstably with a larger linewidth due to the multimode oscillation, mode competition, and modehopping.

Single-longitudinal-mode (SLM) erbium-doped fiber (EDF) lasers are an attractive optical source for generating dualwavelengths owing to their wide gain bandwidth and high extinction ratio [6-10].

Manuscript received September 01, 2014. This work was supported by the Spanish Ministerio de Ciencia y Tecnologia through project TEC2013-47264$\mathrm{C} 2-2-\mathrm{R}$ and the INTERREG SUDOE Project "ECOAL-MGT-Ecological Management of Coal Waste Piles, SOE3/P2/P714". The authors are with the Department of Electrical and Electronic Engineering, Public University of
Several approaches have been proposed to achieve single longitudinal mode (SLM) operation [11-14]. For example, a multi-ring cavity is proposed to guarantee a SLM operation in $[12,13]$. In [13] a SLM fiber-ring laser is achieved by using a saturable absorber. Nevertheless, the efficiency of the fiber laser is reduced with this technique. A fiber Bragg grating Fabry-Pérot etalon can be also used to obtain SLM fiber lasers [14], but the spacing between the different longitudinal modes must be as big as possible to achieve SLM operation. Recently, have been used with photonic crystal fibers (PCFs). For example, a Sagnac reflector with $25 \mathrm{~m}$ PCF into a ring cavity was used in [15]. In [16] a PCF section was used as a functional component inside the ring cavity, whereas in [17] the PCF was used to generate four-wave mixing (FWM). An important feature of MEDRFLs is the optical signal-to-noise ratio (OSNR). In $[18,19]$ a MEDFL with OSNRs as high as $65 \mathrm{~dB}$ and power instabilities around $1 \mathrm{~dB}$ was achieved using FBGs.

In this paper, a stable SLM fiber ring laser is proposed and demonstrated. Optical feedback introduced into the structure effectively reduces mode competition. This suggested scheme operates in SLM regime without needing a precise control of the laser cavity length or including ultra-narrow bandpass filters. The combined interactions of the self-injection feedback and the internal lasing injection provide the longitudinal-mode restriction and guarantee stable SLM laser oscillation. With the proposed feedback fiber loop structure, single-longitudinalmode multi-wavelength lasing can be achieved by using fiber Bragg gratings (FBGs) inside the ring cavity as wavelength selectors. The proposed scheme was validated for temperature sensing, showing a clear linear behavior. Finally, it was checked the applicability of this system for remote sensing nevertheless in this configuration SLM regime cannot be achieved due to the extremely long cavity.

\section{EXPERIMENTAL SETUP}

\section{A. Experimental characterization of the ring laser}

In this work, an EDFRL that uses a feedback path for achieving SLM oscillation is proposed and demonstrated. Far for the simplicity of the scheme, the inclusion of the feedback implies complex interactions that result in an increase of the laser's performance.

The main ring cavity is composed of a 980/1550 nm wavelength division multiplexer used to introduce $100 \mathrm{~mW}$

Navarra, Edificio de los Tejos, Pamplona E-31006, Spain (e-mail: silvia.diaz@unavarra.es; daniel.leandro@unavarra.es; mla@unavarra.es). Copyright (c) 2013 IEEE. Personal use of this material is permitted. 
from a $980 \mathrm{~nm}$ pump laser diode, a $7 \mathrm{~m}$-long section of highly erbium-doped fiber (Er-30 provided by Liekki, with an absorption of $10.8 \mathrm{~dB} / \mathrm{m}$ at $978 \mathrm{~nm})$ acts as the active medium, a 90:10 optical coupler, an optical circulator, a $2 \times 4$ optical coupler and four reflective FBGs. The FBGs determine the lasing wavelength of the EDFRL. The secondary ring includes a variable optical attenuator that is connected to the main ring cavity by the $2 \times 4$ optical coupler. The fiber Bragg gratings used $\lambda_{1}, \lambda_{2}, \lambda_{3}$ and $\lambda_{4}$, correspond to the wavelength values of 1537 , 1545,1550 and $1555 \mathrm{~nm}$ respectively. Variable attenuators were also used before each FBG, but it was hardly necessary to use them; this is one of the main advantages of this system.

An Optical Spectrum Analyzer (OSA) is used to monitor the $10 \%$ output of the ring laser. There is no need to use an isolator, because the 3-port circulator keeps the ring laser operating unidirectionally preventing undesired effects as the spatialhole-burning.

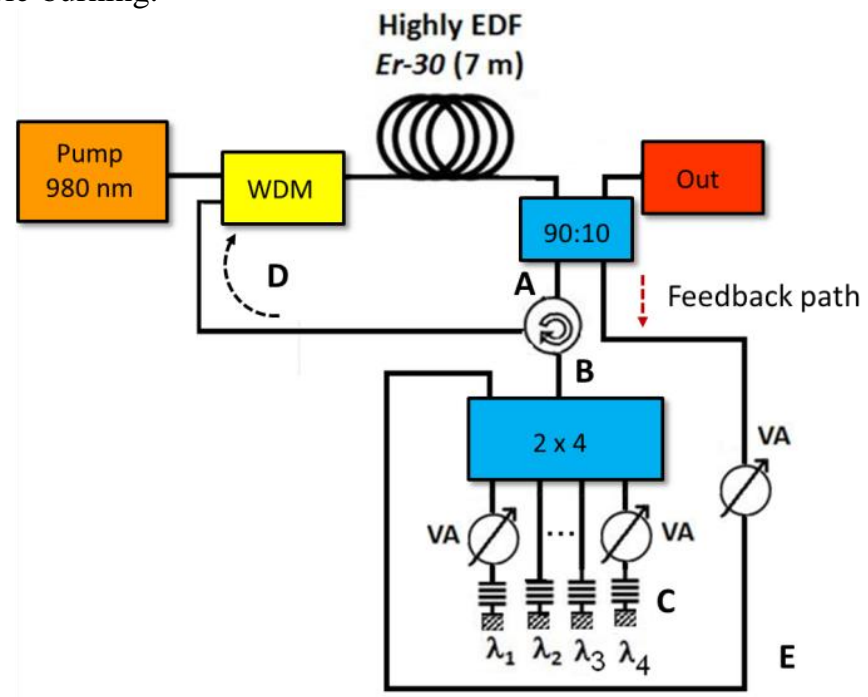

Fig. 1. Schematic diagram for the proposed single-longitudinal mode fiber ring laser [20].

The operating principle of the system relies on two main effects: The first leading effect of the feedback is that two ring cavities of different lengths are generated. The main cavity includes the paths $\mathrm{A} \rightarrow \mathrm{B} \rightarrow \mathrm{C} \rightarrow \mathrm{B} \rightarrow \mathrm{D} \rightarrow \mathrm{A}$ with a total length of $17 \mathrm{~m}$. The secondary ring, which operates co-directionally with the main ring, involves the paths $\mathrm{E} \rightarrow \mathrm{C} \rightarrow \mathrm{B} \rightarrow \mathrm{D} \rightarrow \mathrm{E}$ with a total length of $18 \mathrm{~m}$. Taking into account that the free spectral range (FSR) for a given length is:

$$
F S R=\frac{c}{n L}
$$

where $n$ is the refractive index of the fiber, $c$ is the speed of light in vacuum and $L$ is the length of the cavity. According to this, the FSR obtained for the two cavities are 11.4 and $12.1 \mathrm{MHz}$ for the main and secondary cavities respectively. The full width at half maximum (FWHM) of the FBGs is around $0.24 \mathrm{~nm}$. Additionally, a secondary effect arises due to the feedback path. That is the interference that occurs between the counterpropagating light reflected at the FBGs following the path $\mathrm{C} \rightarrow \mathrm{E}$ and the co-propagating light coming from $\mathrm{C} \rightarrow \mathrm{B} \rightarrow \mathrm{D}$. Due to the Vernier effect mode suppression is attained [21] resulting in fewer longitudinal modes falling within the reflectivity envelope of each grating. Therefore a higher FSR implies that SLM operation can be obtained more easily. According to [22] the two main advantages of including a short optical feedback path are: (1) A considerable spectral narrowing. (2) A more stable laser operation.

In order to study the effect of this feedback fiber loop, a comparison of the performance of the setup with and without that feedback loop was made. Additionally, the application of the laser as a sensing system was also tested.

\section{B. Remote utilization of the laser system for sensing}

To check the applicability of this system for remote sensing, we propose a fiber ring laser remote sensing system. Fig.2 shows the setup, where it is included a fiber lead of $50 \mathrm{~km}$ of standard single-mode fiber after the three-port circulator. This fiber spool simulates a $25 \mathrm{~km}$ long remote system. In this way, the light through the main circulation path and from the feedback path is coupled into the ring. As in the previous case, the spectrum reflected from the sensors is amplified by a $7 \mathrm{~m}$ long EDF before it is directed to the ring. The output of the laser is monitored by an OSA, which is connected to the $10 \%$ port of a 90:10 coupler.

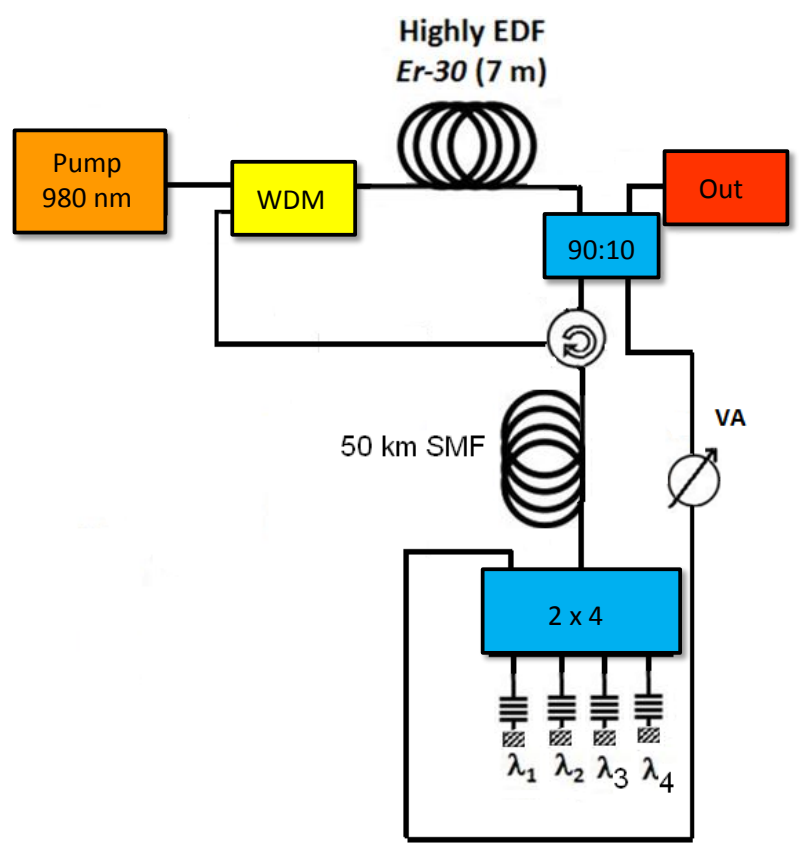

Fig. 2. Schematic diagram of the proposed multi-wavelength erbium-doped fiber ring laser configuration for remote sensing.

\section{EXPERIMENTAL RESULTS}

\section{A. Experimental characterization of the ring laser}

The output spectrum of the proposed multi-wavelength fiber ring laser scheme is shown in Fig. 3(a). This one corresponds to the reflection bands of the four FBGs when the pump power was $100 \mathrm{~mW}$ and when the power in both lasing circulation path and feedback path was equalized. The power obtained from the four channels was around $-26 \mathrm{dBm}$, giving OSNRs higher than $50 \mathrm{~dB}$. 
In order to compare the behavior of the system with and without the feedback, the output power instability of the MEDFRL was measured. The MEDRFL with all the four channel laser outputs in operation was tested for a period of 10 min, as shown in Fig. 3(b). The best instability was $0.212 \mathrm{~dB}$ and corresponds to the FBG centered at $1537 \mathrm{~nm}$. The rest of the measured values were $0.556 \mathrm{~dB}, 0.278 \mathrm{~dB}$ and $0.291 \mathrm{~dB}$, corresponding to the FBGs placed at $1545 \mathrm{~nm}, 1550 \mathrm{~nm}$ and $1555 \mathrm{~nm}$, respectively. This results show that a good stability can be achieved when the feedback is applied to the system.
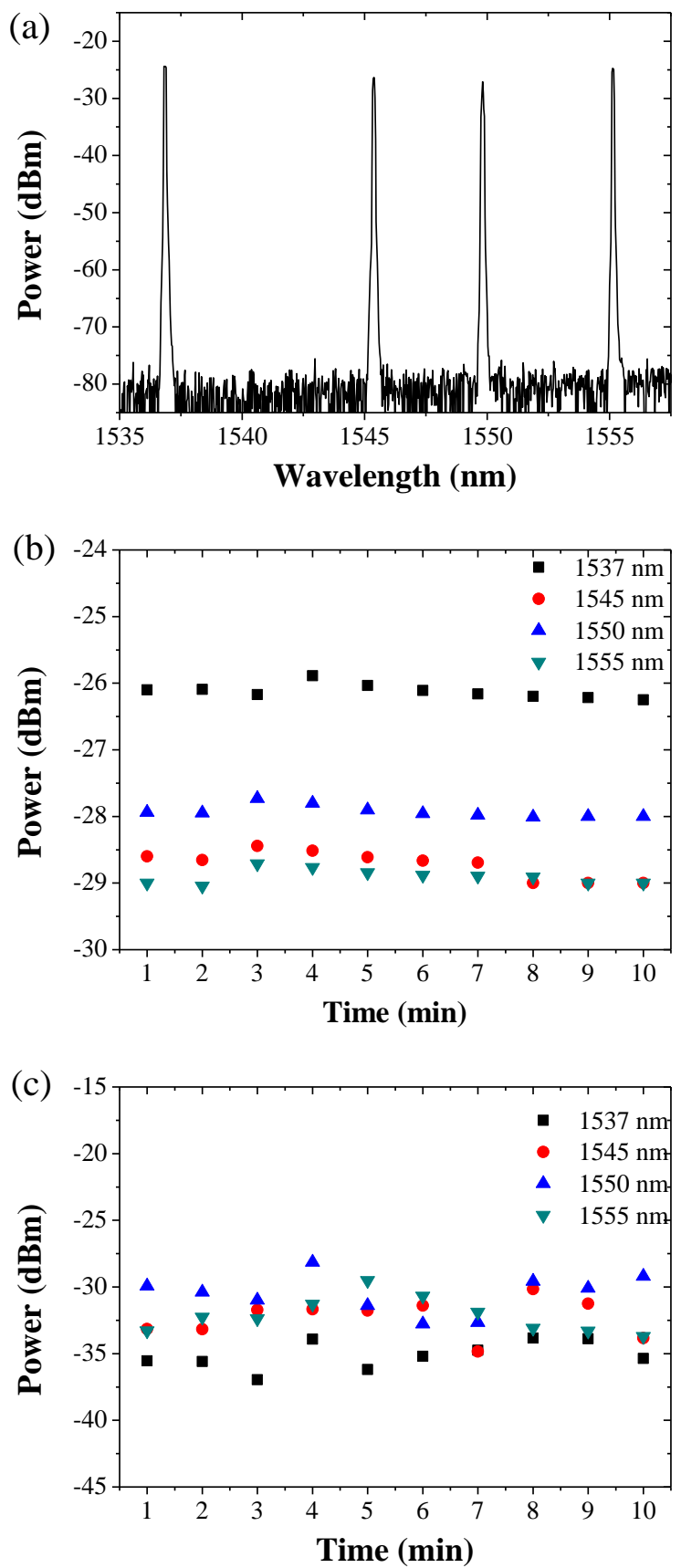

Fig. 3. Output optical spectrum measured by the OSA for the proposed MEDFRL for a pump power of $100 \mathrm{~mW}$ (a) and Output power fluctuation of the four channel lasers with (b) and without (c) feedback.

In contrast, without the feedback path it was almost not possible to equalize the FBGs and the stability results were worse as shown in fig. 3 (c). In this case, the measured stability values were $3,118 \mathrm{~dB}, 4,68 \mathrm{~dB}, 3,58 \mathrm{~dB}$ and $4,19 \mathrm{~dB}$ for the FBGs at $1537 \mathrm{~nm}, 1545 \mathrm{~nm}, 1550 \mathrm{~nm}$ and $1555 \mathrm{~nm}$.

According to the results, the feedback path gives stability to the system, as was previously demonstrated in [22]. An interesting conclusion to be drawn from Fig. 3 is that the use of feedback improves the stability and the OSNR of the system. This is a crucial advantage over some previously reported lasers in the literature [11,23].

When the feedback path and the lasing circulation path present the same output power, the laser behaves with SLM operation (as shown in Fig. 4). This is made by using a VA in the feedback path to adjust the cavity losses in both paths.

In order to demonstrate the SLM behavior a heterodyne technique was used. In a worst case scenario, supposing the longest possible length $(18 \mathrm{~m})$ for a simple cavity, the mode spacing would be $\approx 12 \mathrm{MHz}$. Therefore the beating signal generated between one emission line each time and a tunable laser $(100 \mathrm{kHz}$ linewidth $)$ was detected using a $6 \mathrm{GHz}$ photodetector and an electrical spectrum analyzer (ESA). The tunable laser was set close to one emission line each time with an ESA resolution of $100 \mathrm{kHz}$. Figure 4 shows the four superimposed spectra of the beating of the emission lines, showing a clear SLM behavior.

Finally the system was tested as temperature sensor. Therefore the wavelength of the emission lines was studied versus temperature variations. In this way, one FBG was placed inside a climatic chamber and temperature cycles from $25^{\circ} \mathrm{C}$ to $70^{\circ} \mathrm{C}$ with a $5^{\circ} \mathrm{C}$ step were performed. In Fig. 5, the wavelength increment was approximately $9 \mathrm{pm} /{ }^{\circ} \mathrm{C}$ for the emission line placed at $1537 \mathrm{~nm}$ and an error factor $\mathrm{R}^{2}=0.958$. This indicates that this structure behaves properly and measures correctly temperature variations, as expected. After checking the proper operation of the system as a temperature sensor by using the wavelength of the emission lines, a study of the wavelength instability was carried out. Figure 6 (a) and (b) show the wavelength variation every minute for one hour. The instabilities attained varied between $0.06 \mathrm{~nm}$ and $0.08 \mathrm{~nm}$.

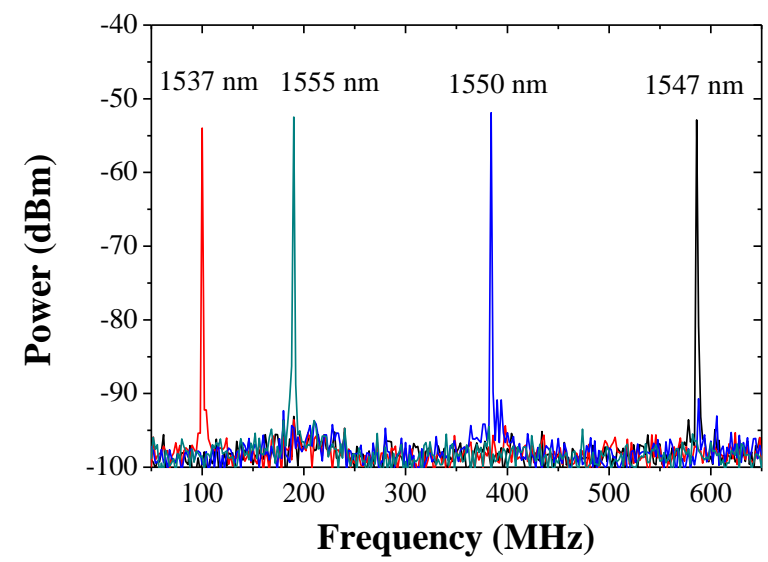

Fig. 4. Superimposed spectra of the beating electrical signal obtained from the heterodyne detection of an emission line each time with a tunable laser. 


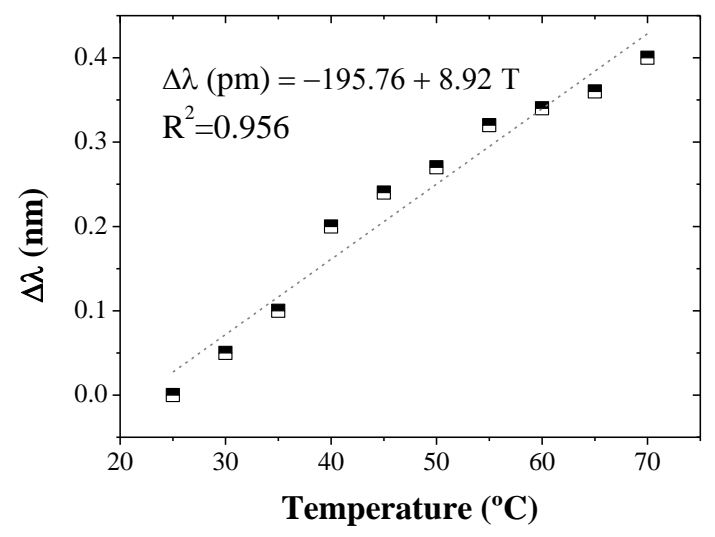

Fig. 5. Laser output spectrum from the four sensors.
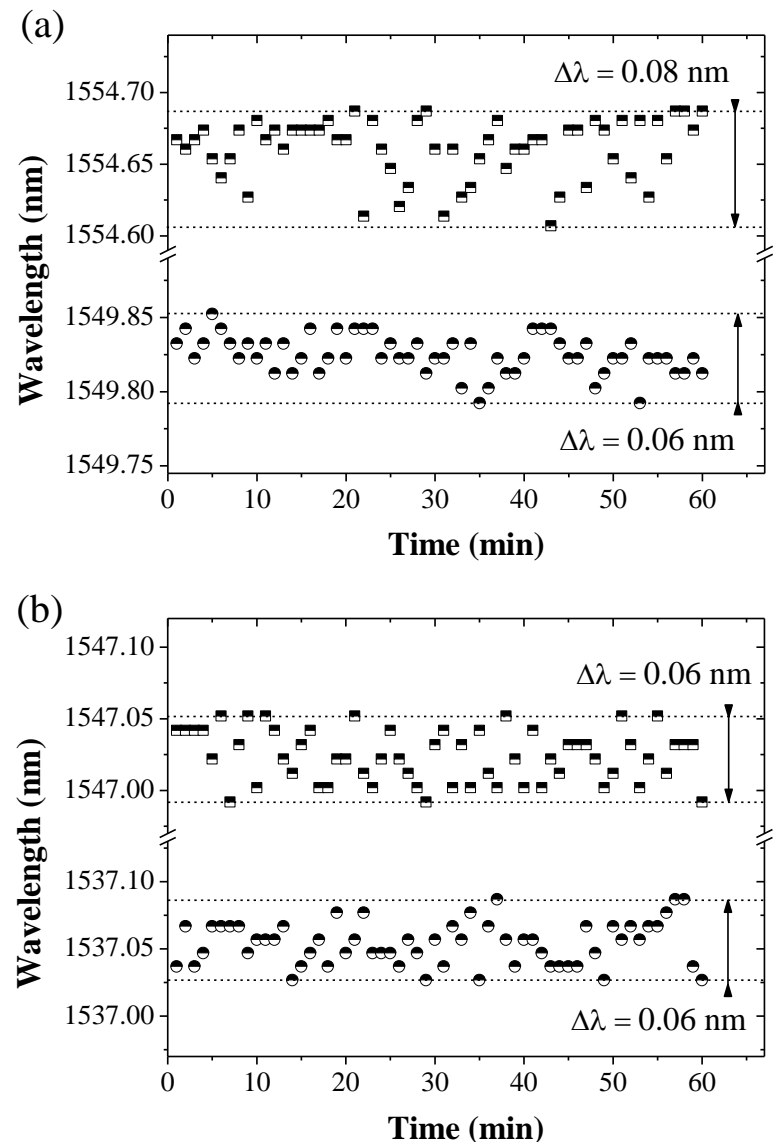

Fig. 6. Wavelength fluctuation of the emission lines placed at (a) 1554.6, 1549.8 , (b) 1547 and $1537 \mathrm{~nm}$.

Finally, it was determined the emission lines bandwidth using a self-heterodyne method based on [23]. Fig. 7(a) and (b) show the spectrum at the ESA with a resolution of $1 \mathrm{kHz}$ for the FBGs at $1545 \mathrm{~nm}$ and $1555 \mathrm{~nm}$, respectively. As reported previously in literature due to the Gaussian fitting, this spectrum was $\sqrt{2} \mathrm{BW}$, where $\mathrm{BW}$ means the laser bandwidth [24]. The BW for the FBG centered at $1545 \mathrm{~nm}$ and $1555 \mathrm{~nm}$ were $2.85 \mathrm{KHz}$ and $3.25 \mathrm{kHz}$, respectively. The remaining values were, 3.62 and $3.79 \mathrm{kHz}$, for the FBGs at $1537 \mathrm{~nm}$ and $1550 \mathrm{~nm}$, respectively.
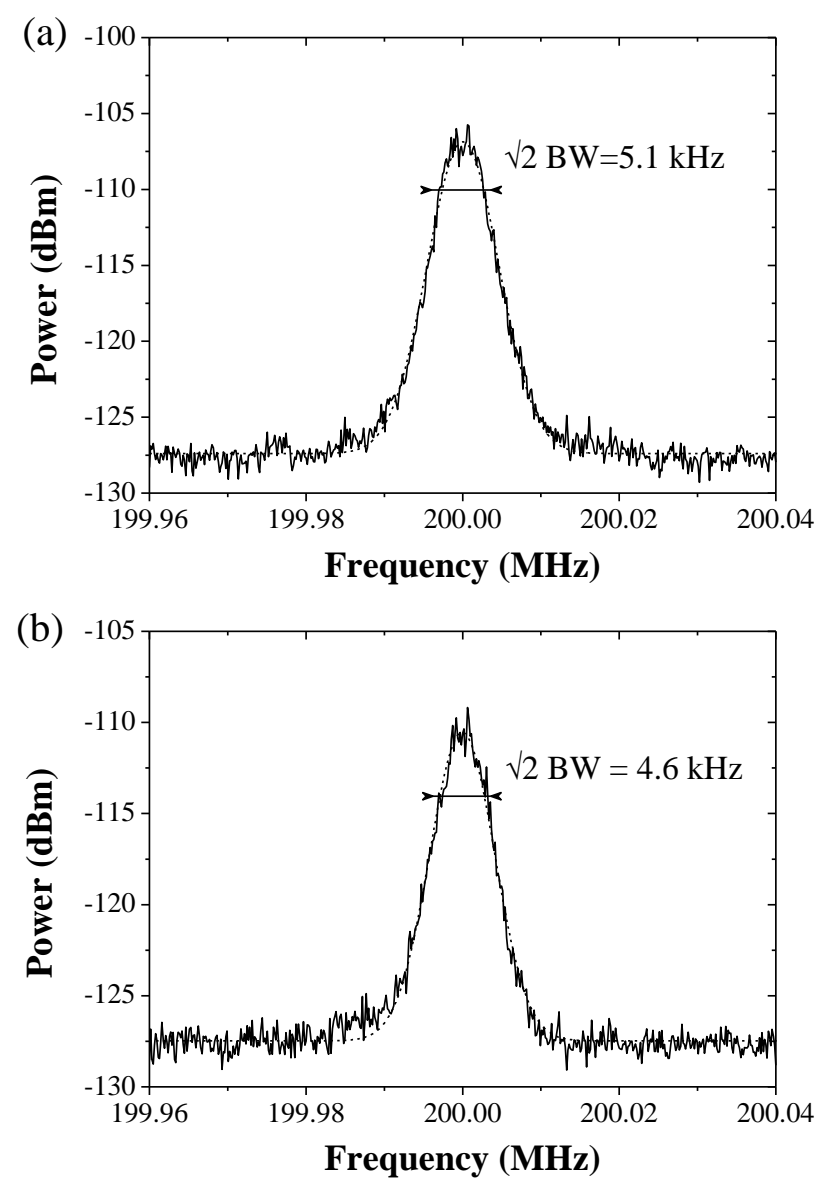

Fig. 7. Spectrum of the emission line measured at ESA for the FBG at 1545 $\mathrm{nm}$ (a) and for the FBG at $1555 \mathrm{~nm}$. (b)

As proposed in Section II A, the experimental results probed that the optical feedback provides two important advantages for lasers operating in SLM. The first one is an increase in the power stability. The second implies a remarkable linewidth narrowing. In this case, in order to compare the cases with and without feedback, the linewidths of the emission lines could not be measured without feedback since it was not possible to obtain a stable condition. It is worth mentioning that the main improvement of the feedback is that allows the system to work in a stable regime which is not a measurable progress.

\section{B. Remote utilization of the laser system for sensing}

Fig.8 shows the output spectrum of the proposed remote multi-wavelength fiber ring laser scheme corresponding to the reflection bands of the four FBGs when the pump power was $200 \mathrm{~mW}$ and when the power in both lasing circulation path and optical path was equalized. The measured power of the four channels was around $-38 \mathrm{dBm}$, obtaining SNRs higher than 17 dB. 


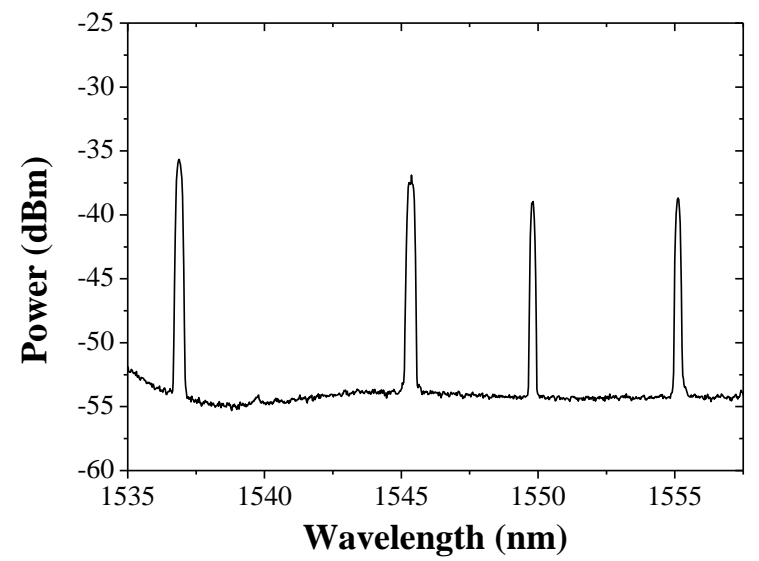

Fig. 8. Laser output spectrum from the four sensors.

To demonstrate the remote-sensing ability of the system, we performed temperature measurement with it. Because of the length of the new structure, the single-mode behavior of the laser is lost. However, a good OSNR is still achieved, enough for remote measurements. As mentioned before, an available 50 $\mathrm{km}$ fiber reel was used to simulate a $25 \mathrm{~km}$ remote measurement. Because of the non-uniform ASE spectrum of the $\mathrm{EDF}$, the output power of the laser changes with the temperature. To detect the wavelength shift of the FBGs in response to a temperature change, we place one of the reflective FBGs into a climatic chamber, in which case the lasing wavelength provides a measure of the temperature. The remaining components of the setup is kept at room temperature. Again, the chamber temperature is adjusted from $25^{\circ} \mathrm{C}$ to $70^{\circ} \mathrm{C}$ with a $5^{\circ} \mathrm{C}$ step.

Fig. 9 shows the variation of the $1537 \mathrm{~nm}$ wavelength according to the chamber temperature. It can be clearly seen that the change of the lasing wavelength is linear to the temperature change. The temperature sensitivity of the sensor is $9.4 \mathrm{pm} /{ }^{\circ} \mathrm{C}$ for the $\mathrm{FBG}$ of $1537 \mathrm{~nm}$, which indicates that this structure measures correctly temperature variations.

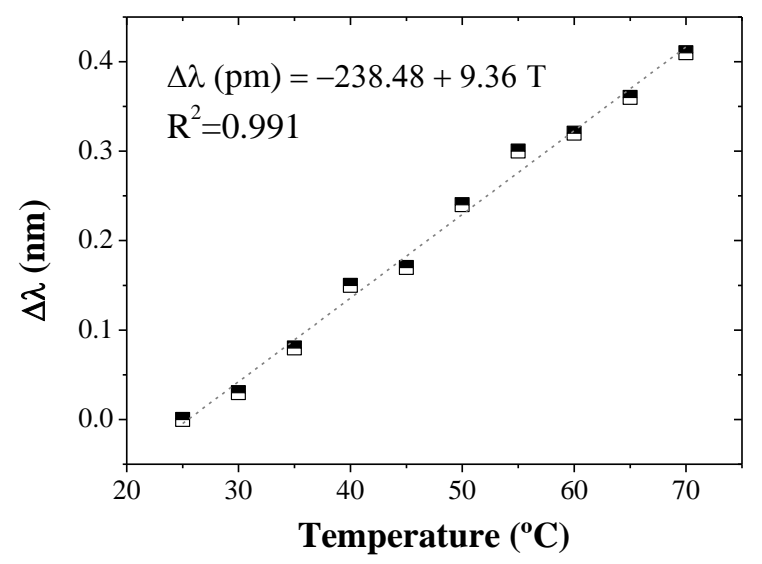

Fig. 9. Variation of the lasing wavelength with the sensor temperature.

\section{CONCLUSIONS}

A novel configuration of a stable MEDFRL based on optical feedback has been demonstrated. The laser employs four fiber Bragg gratings to select the operation wavelengths and as sensing elements. By equalizing the power of the lasing circulation path and the feedback path the laser operates in SLM regime. The inclusion of the feedback path generates a double co-propagating ring cavity combined with a counterpropagating signal, increasing the FSR. The power instability improvement has been measured, obtaining an average instability of $0.5 \mathrm{~dB}$ with feedback instead of the $4 \mathrm{~dB}$ obtained without it. The linewidth of the emission lines has been also measured obtaining values between 2.85 and $3.79 \mathrm{kHz}$. It is worth noticing that the main enhancement of the system is that without the feedback path the equalization of the emission lines is not possible. Finally, the application of this system for remote temperature measurements has been demonstrated. Our experimental sensor shows a sensitivity of $9.4 \mathrm{pm} /{ }^{\circ} \mathrm{C}$ over the temperature range from 25 to $70^{\circ} \mathrm{C}$ with a sensing distance is about $25 \mathrm{~km}$.

\section{ACKNOWLEDGMENT}

We thank Liekki for providing the Er-doped fibers.

\section{REFERENCES}

[1] R.A. Perez-Herrera, S. Chen, W. Zhao, T. Sun, K.T.V. Grattan, and M. Lopez-Amo, "Stability performance of short cavity Er-doped fiber lasers," Opt. Commun., vol. 283, pp. 1067-1070, March 2010.

[2] Y. Y. Zhu, W. L. Zhang, and Y. Jing, "Tunable Multi-Wavelength Fiber Laser Based on Random Rayleigh Back-Scattering," IEEE Photon. Tehnol. Lett., vol. 25, pp. 1559-1561, Aug. 2013.

[3] H. Chen, "Multiwavelength fiber ring lasing by use of a semiconductor optical amplifier," Opt. Lett., vol. 30, pp. 619-621, March 2005.

[4] H. Dong, G. Zhu, Q. Wang, H. Sun, N. K. Dutta, J. Jaques, and A. B. Piccirilli, "Multiewavelength fiber ring laser source based on a delayed interferometer," IEEE Photon. Technol. Lett., vol. 17, pp. 303-305, Feb. 2005.

[5] C. H. Yeh, C. W. Chow, Y. F. Wu, Y. H. Lin, B. C. Cheng, and J. H. Chen, "Using optimal cavity loss and saturable-absorber passive filter for stable and tunable dual-wavelength erbium fiber laser in singlelongitudinal-mode operation," Laser Phys. Lett., vol. 8, pp. 672-677, July 2011.

[6] S. Diaz, M. Lopez-Amo, "Dual-wavelength single-longitudinal-mode erbium fiber laser for temperature measurements," Opt. Eng., vol. 53, pp. 036106, March 2014

[7] H. B. Sun, X. M. Liu, Y. K. Gong, X. H. Li, and L. R. Wang, "Broadly tunable dual-wavelength erbium-doped ring fiber laser based on a highbirefringence fiber loop mirror," Laser Phys., vol. 20, pp. 522-527, Dec. 2009.

[8] J. E. Im, B. K. Kim, and Y. Chung, "Tunable single-and dual-wavelength erbium-doped fiber laser based on Sagnac filter with a high-birefringence photonic crystal fiber," Laser Phys., vol. 21, pp. 540-547, Feb. 2011.

[9] T. Wang, G. Liang, X. Miao, X. Zhou, and Q. Li, "Stable dual-wavelength single-longitudinal-mode ring erbium-doped fiber laser for optical generation of microwave frequency," Laser Phys., vol. 22, pp. 948-952, Apr. 2012.

[10] S. Feng, O. Xu, S. Lu, T. Ning, and S. Jian, "Switchable singlelongitudinal-mode dual-wavelength erbium-doped fiber ring laser based on one polarization-maintaining fiber Bragg grating incorporating saturable absorber and feedback fiber loop," Opt. Commun., vol. 282, pp. 2165-2168, Jun. 2009.

[11] M.A. Quintela, R.A. Perez-Herrera, I. Canales, M. Fernandez-Vallejo, M. Lopez-Amo, J.M. Lopez-Higuera, "Stabilization of dual-wavelength 
erbium-doped fiber ring lasers by single-mode operation," IEEE Photonics Technol. Lett., vol. 22, pp. 368-370, March 2010.

[12] S. Pan, Z. Xiaofan, L. Caiyun, "Switchable single-longitudinal-mode dual-wavelength erbium-doped fiber ring laser incorporating a semiconductor optical amplifier," Opt. Lett., vol. 33, pp. 764-766, Apr. 2008.

[13] K. Zhang, and J.U. Kang, "C-band wavelength-swept single-longitudinalmode erbium-doped fiber ring laser," Opt. Express, vol.16, pp. 1417314179, Aug. 2008.

[14] J. L. Zhou, L. Xia, X. P. Cheng, X. P. Dong, and P. Shum, "Photonic generation of tunable microwave signals by beating a dual-wavelength single longitudinal mode fiber ring laser," App. Phys. B, Lasers Opt., vol. 91, pp. 99-103, Feb. 2008.

[15] D. Chen, "Stable multi-wavelength erbium-doped fiber laser based on a photonic crystal fiber Sagnac loop filter," Laser Phys. Lett., vol. 4, pp. 437-439, Jun 2007.

[16] D. Chen, and L. Shen, "Switchable and tunable Erbium-doped fiber ring laser incorporating a birefringent and highly nonlinear photonic crystal fiber," Laser Phys. Lett., vol. 4, pp. 368-370, May 2007.

[17] X. Liu, X. Zhou, X. Tang, J. Ng, J. Hao, T. Y. Chai, E. Leong, and C. Lu, "Switchable and tunable multiwavelength erbium-doped fiber laser with fiber Bragg gratings and photonic crystal fiber," IEEE Photon. Technol. Lett., vol. 17, pp. 1626-1628, Aug. 2005.

[18] S. Rota-Rodrigo, R. A. Perez-Herrera, M. Fernandez-Vallejo, M. LopezAmo, "Low noise dual-wavelength erbium fiber laser in singlelongitudinal-mode operation," Appl. Phys. B, vol. 106, pp. 563-567, Jan. 2012.

[19] S. Rota-Rodrigo, I. Ibañez, and M. Lopez-Amo, "Multi-wavelength fiber laser in single-longitudinal mode operation using a photonic cristal fiber Sagnac interferometer," Appl. Phys. B, vol. 110, pp. 303-308, Jan. 2013.

[20] S. Diaz, D. Leandro, and M. Lopez-Amo, "Multi-wavelength erbium fiber ring laser with optical feedback for temperature measurements," in Proc. SPIE vol.9157, 23rd Int. Conf. on Opt. Fibre Sensors, 2014, pp. 91575 Y.

[21] P. Urquhart, "Compound optical-fiber-based resonator," J. Opt. Soc. Am. A, vol. 5, pp. 803-812, June 1988.

[22] Y. Zhao, C. Shu, S.P. Li, H. Ding, K.T. Chan, "Multiple wavelength operation of a unidirectional Er-doped fiber ring laser with optical feedback," in Proc. CLEO vol. 11, 1997, pp. 396.

[23] L. Talaverano, S. Abad, S. Jarabo, and M. Lopez-Amo, "Multiwavelength fiber laser sources with Bragg-grating sensor multiplexing capability," $J$. Lightwave Technol., vol. 9, pp. 553-558, Apr. 2001.

[24] T. Okoshi, K. Kikuchi, and A. Nakayama, "Novel method for high resolution measurement of laser output spectrum," Electron. Lett., vol. 16, pp. 630-631, June 1980.

S. Diaz received the telecommunications engineering and $\mathrm{Ph} . \mathrm{D}$. degrees from the Public University of Navarra, Spain, in 2002 and 2007, respectively. In October 2003, she was a visiting Ph.D. student at the Photonics Group in University of Cantabria, Spain, working on signal processing with distributed fiber optic networks.

In November 2004, she became an Assistant Professor at the Electrical and Electronic Engineering department of the Public University of Navarra. During the summer of 2006, she was a visiting $\mathrm{Ph} . \mathrm{D}$. student at the NAM Laboratory at the École Polytéchnique Fédérale of Lausanne, Switzerland, working on Brillouin Scattering. In 2009, she was a visiting Research Fellow at the University of Bath, UK. Currently, she is an Associate Professor at the Electrical and Electronic Engineering Department of the Public University of Navarra. Her research interests are in distributed non linear amplified networks for optical fiber sensors, multiwavelength fiber lasers, Raman amplifiers, erbium-doped amplifiers and their applications in wavelength-division-multiplexing communication systems and networks.

D. Leandro was born in Pamplona, Spain, in November 1984. $\mathrm{He}$ received the telecommunication engineering degree and communication master degree from the Universidad Publica de Navarra, Spain in 2010 and 2012, respectively. In 2012, he joined the Optical Communications Group, Department of Electrical and Electronic Engineering from Universidad Publica de Navarra. He has been a visiting Ph.D. student at the School of Engineering and Mathematical Sciences at City University of London. His research interests are in fiber optic lasers, optical amplifiers, optical fiber sensor networks and multiplexing architectures.

M. Lopez-Amo (M'91, SM'98) was born in Madrid, Spain, in 1960. He received the M.Sc. telecommunication engineering degree and the Ph.D. degree from the Universidad Politécnica de Madrid, Spain in 1985 and 1989, respectively. From 1985 to 1996, he was with the Photonic Technology Department, Universidad Politécnica de Madrid, where in 1990 he became an Associate Professor.

In 1996, he moved to the Public University of Navarra (Pamplona, Spain) where he became a Full Professor in the Electrical and Electronic Engineering Department and is currently the Head of the Optical Communications Group of this department. He has been Chairman of the Optoelectronic Committee of Spain. He has been leader of more than 30 research projects and he has coauthored more than 250 works in international refereed journals and conferences related with fiber-optic networks, optical amplifiers, fiber-optic sensors, and integrated optics. He is a member of the technical committees of the International Conference on Fiber Optic Sensors (OFS), the European Workshop on Optical Fiber Sensors (EWOFS), and European Cost TD1001 action, among others. Prof. LópezAmo is senior member of the IEEE and member of the OSA. 